\title{
Recent Progress of the Project of the Chinese Spallation Neutron Source
}

\author{
J. ZHANG ${ }^{a}, *$, Q.W. YAN ${ }^{a}$, C. ZHANG ${ }^{\text {b }}$, P.L. ZHANG ${ }^{a}$, S.N. FU ${ }^{b}$, F.W. WANG ${ }^{a}$, \\ Z. ZHANG ${ }^{a}$ and S.X. FANG ${ }^{b}$ \\ a Institute of Physics, CAS, P.O. Box 603, Beijing 100080, People's Republic of China; \\ bInstitute of High Energy Physics, CAS, P.O. Box 918, Beijing 100039, People's Republic \\ of China
}

We report on the recent progress of the project of the Chinese Spallation Neutron Source. The preliminary conceptual designs of the accelerator complex, the target system and the neutron scattering instruments are included.

Keywords: CSNS project; Conceptual design; Accelerator; Target station; Neutron spectrometers

\section{INTRODUCTION}

The proposal of the Chinese Spallation Neutron Source (CSNS) project was granted in the beginning of 2002 after three review meetings, organized by the Chinese Academy of Sciences (CAS) and other scientific organizations. Physicists from the Institute of Physics (IP) and the Institute of High Energy Physics (IHEP), both belonging to CAS, consequently started a conceptual design and feasibility study. The CSNS plan calls for a 70- $\mathrm{MeV} \mathrm{H}^{-}$linac and a $1.6 \mathrm{GeV}$ rapid cycling synchrotron (RCS) producing a proton current of $62.5 \mu \mathrm{A}$ $(100 \mathrm{~kW})$ at a $25 \mathrm{~Hz}$ repetition rate. It should be able to be upgraded to a higher beam power in its second phase. The CSNS target station design team, has initiated the conceptual design of the target-moderator system based on the suggestions and comments [1] from an international advisory team, in the first moderator-target planning meeting of CSNS project (April 21-26, 2002 in Beijing). In consideration of the characteristics of the spallation neutron source, the budgets and possible requests for future users in China, four multipurpose neutron scattering spectrometers were proposed to be constructed as the first step. In this present paper, we describe recent progress of the conceptual design and feasibility study on proton acceleration complex, target-moderator system and scattering instruments.

\section{PROTON ACCELERATOR COMPLEX}

The CSNS plan calls for a $70-\mathrm{MeV} \mathrm{H}^{-}$linac and a $1.6 \mathrm{GeV}$ synchrotron producing a proton current of $62.5 \mu \mathrm{A}(100 \mathrm{~kW})$ at a $25 \mathrm{~Hz}$ repetition rate. The design goal of the CSNS is listed in Table I and the schematic layout of the CSNS is shown in Fig. 1.

*Corresponding author. Fax: + 86-10-8264-9531. E-mail: jzhang@ aphy.iphy.ac.cn 
TABLE I The design goal of the CSNS

\begin{tabular}{lcc}
\hline Item & Unit & Value \\
\hline RFQ injection energy & $\mathrm{keV}$ & 75 \\
DTL injection energy & $\mathrm{MeV}$ & 3.5 \\
RCS injection energy & $\mathrm{MeV}$ & $70-130$ \\
Beam energy on target & $\mathrm{GeV}$ & 1.6 \\
Repetition of RCS & $\mathrm{Hz}$ & 25 \\
Average beam current & $\mu \mathrm{A}$ & $62.5-125$ \\
Average beam power & $\mathrm{kW}$ & $100-200$ \\
\hline
\end{tabular}

As seen in Fig. 1, the linac is composed of $\mathrm{RF} \mathrm{H}^{-}$ion source, low energy beam transport (LEBT), radio frequency quadrupole accelerator (RFQ), drift tube linac (DTL). The $\mathrm{RF} \mathrm{H}^{-}$ source will operate with the beam current of $20 \mathrm{~mA}$ under the pressure of hydrogen gas less than $20 \mathrm{mTorr}$. The RFQ can produce an $I \sim 40 \mathrm{~mA}$ beam in the average aperture of diameter $\sim 6 \mathrm{~mm}$, the energy reaches $2-5 \mathrm{MeV}$. Then DTL accelerates the beam to the energy of $70-130 \mathrm{MeV}$ with an average beam current $I>100 \mu \mathrm{A}$. The proton beam is injected from the linac into a RCS, and accelerated to $1.6 \mathrm{GeV}$ with beam current of $62.5 \mu \mathrm{A}$. A lattice of a topological 4-folder ring was proposed which includes 16 FODO cells with 32 dipoles in arcs and four long straight sections among arcs. The deflection angle at each dipole is $11.25^{\circ}$. There are, in total, 68 focusing and defocusing quadrupoles along the whole ring.

Some key technologies are being developed in order to meet the design specification of the CSNS accelerators. The construction of a $3 \mathrm{MeV}$ RFQ with duty factor of $6 \%$ is in progress. The new technology with finemet loaded structure is adopted for the RF cavities of the RCS. The rapid cycling magnets and their power supplies are studied. A prototype of White circuit type power converter is underway. To solve the Eddy current problem during the rapid cycling, the ceramic vacuum chambers with titanium-nitrogen coating will be applied for the RCS. The corresponding R and D is carried on. The proton beams are extracted from the RCS by means of a fast kicker and magnetic septa, and them delivered to the target through a high energy beam transport line.

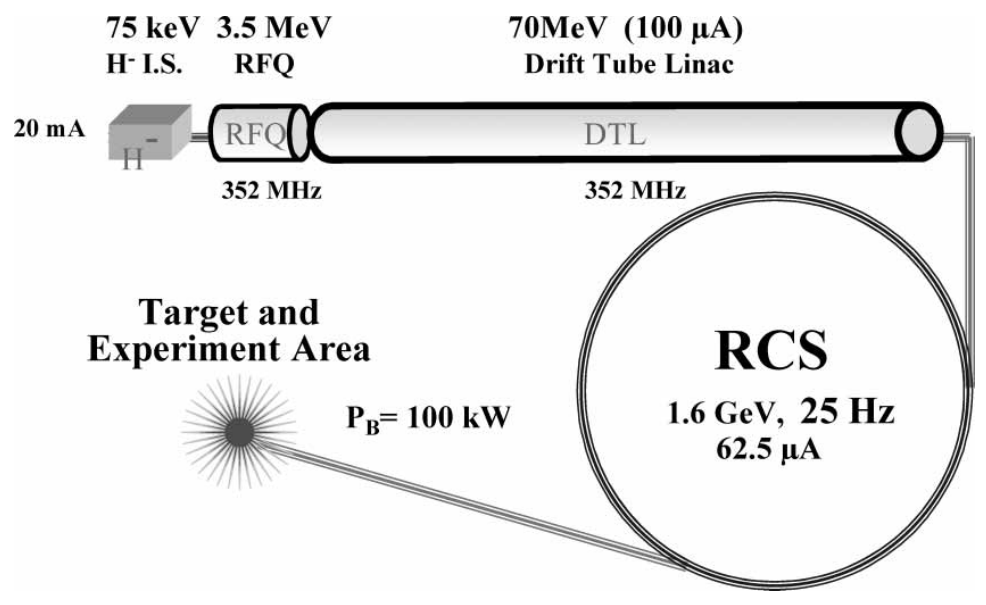

FIGURE 1 A schematic layout of the CSNS. 


\section{TARGET-MODERATOR SYSTEM}

The CSNS target station will be constructed with 40 pieces of tungsten plates with $\mathrm{D}_{2} \mathrm{O}$ as the coolant. Along with the target, there are Beryllium/Iron reflectors (Beryllium: $\phi 1200 \mathrm{~mm}$ and Iron $\phi 1200-2000 \mathrm{~mm}$ ) and Iron/high-density-iron-aggregateconcrete biological shielding $(\phi 2000-10,000 \mathrm{~mm})$. Three wing-mounted moderators: water (room temperature), liquid-methane $(100 \mathrm{~K})$ and liquid-hydrogen $(20 \mathrm{~K})$ and 18 horizontal neutron-apertures with three in front and three behind the moderator.

The designed CSNS target station uses the narrow plate tungsten target, which allows the moderator to be as close as possible to the High-neutron-flux area in the centre of the target. The preliminary target consists of 40 narrow tungsten plates of size, $40 \mathrm{~mm}$ (high) $\times 100 \mathrm{~mm}$ (width) $\times 10 \mathrm{~mm}$ (thickness) for each with a $1.5 \mathrm{~mm}$ gap between each plate enabling coolant flow through for heat dissipation. The Monte Carlo algorithm NMTC/JAM [2], developed by the researchers of JAERI, is used for our simulation. The influence of the Beryllium/Iron reflector and the Iron/high-density-iron-aggregate-concrete is also considered in this simulation. The simulation shows the best height-width ratio of near 1:2.5 of the cross section has the best effect in neutron production [3]. As shown in Fig. 2, all the targets have the same height-width ratio $(1: 2.5)$ in the simulation. One can find that the neutron production increased for about 50\% when the cross section area is reducing from $60 \times 150$ to $40 \times 100 \mathrm{~mm}^{2}$, and that the best length of the tungsten target stack is about $40 \mathrm{~cm}$.

The target cross section is $40 \times 100 \mathrm{~mm}^{2}$, thus the proton beam cross section $32 \times 80 \mathrm{~mm}^{2}$, probably raises the temperature in the target. We simulated the temperature distribution in the target stacks with the coolant velocity of $2 \mathrm{~m} / \mathrm{s}$. Figure 3 shows the results of temperature for the first 15 pieces target plates. The highest temperature reaches almost $92^{\circ} \mathrm{C}$, which may be lowered by a relatively high coolant velocity.

\section{SCATTERING INSTRUMENTS}

It is well known that, as a micro-probe, neutron scattering technique has been and will be widely used in various fields of science and industry, especially recently in the fields of the nano and biological technologies, due to its wide coverage of wavelength and energy. Neutron scattering is used to study the arrangement, motion and interaction of atoms in materials, usually provides valuable information that often cannot be obtained by other techniques, such as optical spectroscopy, electron microscopy and X-ray diffraction. Researchers need all these techniques to provide the maximum amount of information on materials. In consideration of the characteristics of the spallation neutron source, the financial support and the users in China, four multi-purpose neutron scattering spectrometers - the general powder diffractometer (GPD), broad Q-range small angle

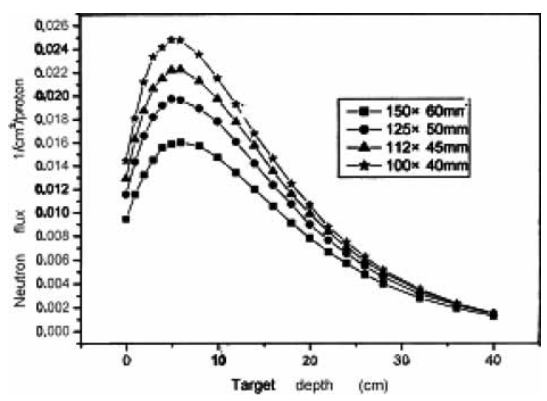

FIGURE 2 Transgress neutron production. 

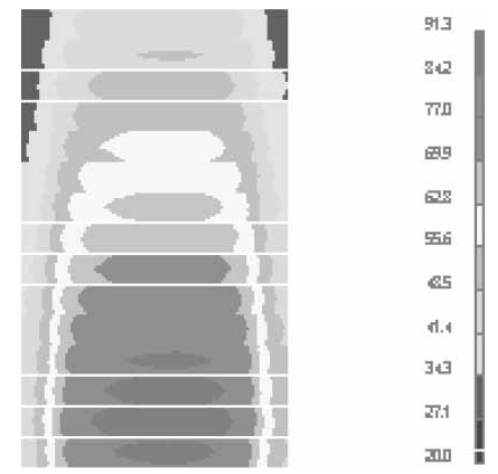

FIGURE 3 The simulated temperature distribution of the first 15 pieces of target plates.

diffractometer (SANS), multi-purpose reflectometer (MPR) and direct geometric inelastic spectrometer (DG-INS) - are proposed to be constructed by CAS as the first step.

Now we are carrying out the Monte Carlo simulation (VITESS and IDEAS) to optimize the design of GPD, then others proposed. In addition, a high resolution quasi-elastic and inelastic neutron scattering spectrometer is also being considered to study the molecular dynamics, rotational and translational diffusion etc.

\section{SUMMARY}

The stage for conceptual design and feasibility study for the CSNS project will be finished by the end of this year. We wish we can have an extensive international collaboration and in turn make our contribution to the society.

\section{Acknowledgement}

This work was supported by the NKBRSE under Grant No. 2002CB713903.

\section{REFERENCES}

[1] Comment of International committee of the first moderator-target planning meeting for CSNS project (2002), April 22-25, Beijing.

[2] Koji Nita, Shin-ichiro Meigo, Hiroshi Takada and Yujiro Ikeda, High Energy Particle Transport Code NMTC/JAM (2001), Tokai-mura: Japan Atomic Energy Research Institute.

[3] Yin, W. and Liang, J.-Q. (2003) "Neutronics studies of solid targets for spallation neutron source using Monte Carlo simulation", Chin. Phys. 12, 599. 